\title{
Assessment of semi-quantitative parameters for visual interpretation of stress-perfusion CMR in obstructive coronary artery disease
}

\author{
Christoph J Jensen ${ }^{1 *}$, Lowie M Van Assche ${ }^{1}$, Deneen Spatz ${ }^{1}$, Michele Parker ${ }^{1}$, Wolfgang G Rehwald ${ }^{2,1}$, \\ Raymond J Kim', Igor Klem ${ }^{1}$ \\ From 16th Annual SCMR Scientific Sessions \\ San Francisco, CA, USA. 31 January - 3 February 2013
}

\section{Background}

Adenosine stress CMR with visual interpretation is increasingly used in the evaluation of patients with CAD. The definition of a stress perfusion defect is inconsistent in the literature regarding i) the duration from contrast arrival, ii) persistence relative to a remote segment, iii) transmural extent, and iv) reversibility on rest perfusion.

In this study we sought to test several semi-quantitative parameters assessed by rapid visual analysis, and determine their utility to identify stress-perfusion defects from obstructive CAD.

\section{Methods}

25 patients (61 \pm 14 years, $44 \%$ male) with known CAD and $\geq 70 \%$ stenosis on invasive coronary angiography (CA) were studied (60\% single-vessel, $40 \%$ two-vessel disease). All patients underwent CMR within 2 months of CA, typically, four short-axis images were obtained each heartbeat during adenosine stress and rest. Patients with infarction, revascularization, and cardiomyopathy were excluded.

Stress and rest perfusion images were analyzed visually using a 16-segment model. The number of frames from left-ventricular (LV) cavity peak contrast to myocardial signal homogeneity (MET) within each segment was assessed ( $n=400$ segments). We scored the transmural extent (in quartiles) of any segmental hypoenhancement persiting $\geq 1$ frame beyond the first homogenous segment in the same slice. CA was reviewed to determine for each segment, whether they

'Duke Cardiovascular Magnetic Resonance Center, Duke University, Durham, NC, USA

Full list of author information is available at the end of the article are subtended by a stenotic coronary artery ("ischemic" vs "non-ischemic" segments).

\section{Results}

The input function (number of frames from LV contrast arrival to $L V$ peak contrast enhancement) ranged between 4-7 frames (median 5, IQR 4-6) in all patients. There were 154 ischemic and 246 non-ischemic segments. Nine non-ischemic segments had a matched rest perfusion defect (e.g. artifactual), none of the ischemic segments showed a rest perfusion defect. MET for ischemic segments was longer (median 22, interquartile range [IQR] 14-30) than in non-ischemic segments (7, IQR 5-8, p<0001), however, 38\% of segments overlapped (Figure 1a). Most non-ischemic segments were concurrently homogenous (0, IQR $0-0)$, in two segments hypoenhancement persisted for $>3$ frames, both had matched rest perfusion defects (Figure 1b). In ischemic segments hypoenhancement persisted for a median of 14 (IQR 8-22) frames $(\mathrm{p}<0.0001)$, with a minimum of 4 frames. The transmural extent of hypoenhancement (Figure 1c) was higher in ischemic segments (3, IQR 2-3) compared to non-ischemic segments (0, IQR 0-0], 139 (90.3\%) ischemic segments were $>25 \%$ transmural.

\section{Conclusions}

Stress-perfusion defects in obstructive CAD tend to be $>25 \%$ in transmural extent, persist for $>4$ frames, and have no corresponding rest perfusion defects.

\section{Funding}

none. 


\section{a)}

\section{Frequency of non-ischemic and ischemic segments according to the myocardial enhancement time}

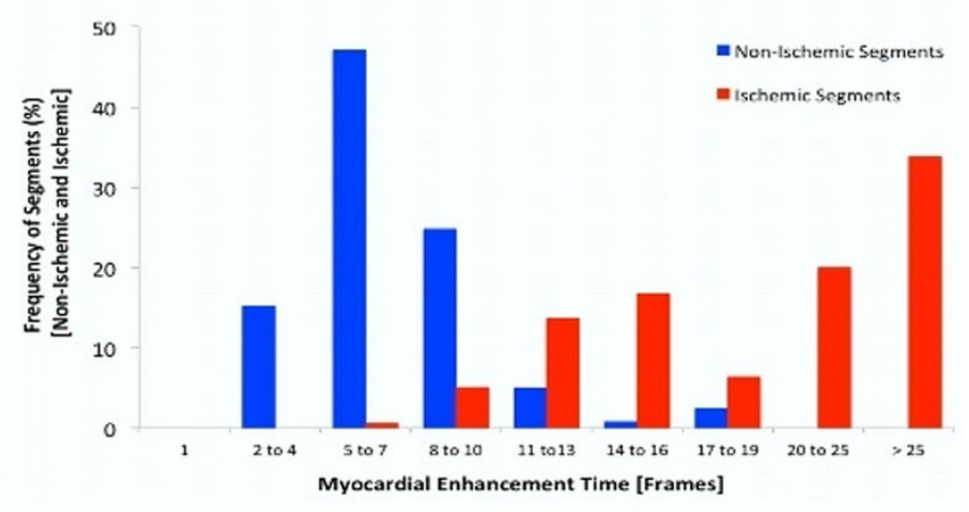

b)

Frequency of non-ischemic and ischemic segments according to the persistence of hypoenhancement

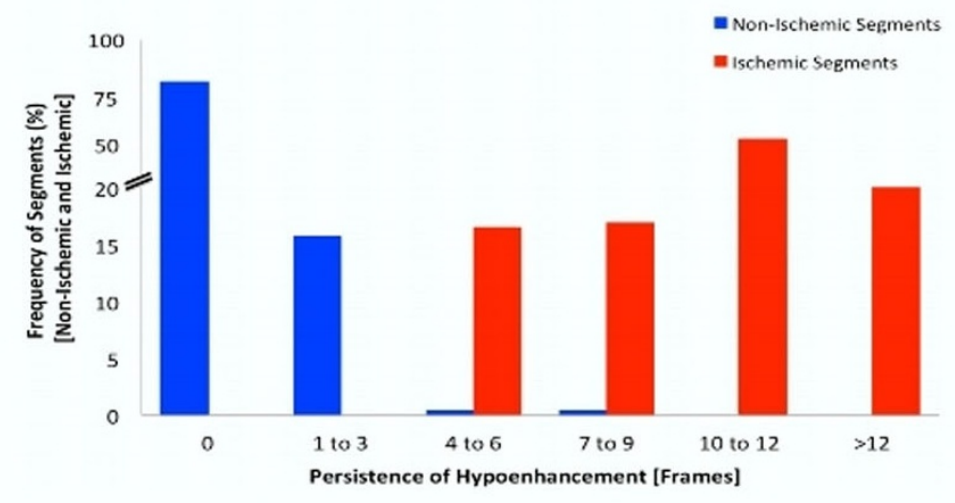

c)

Frequency of non-ischemic and ischemic segments according to the transmural extent of hypoenhancement

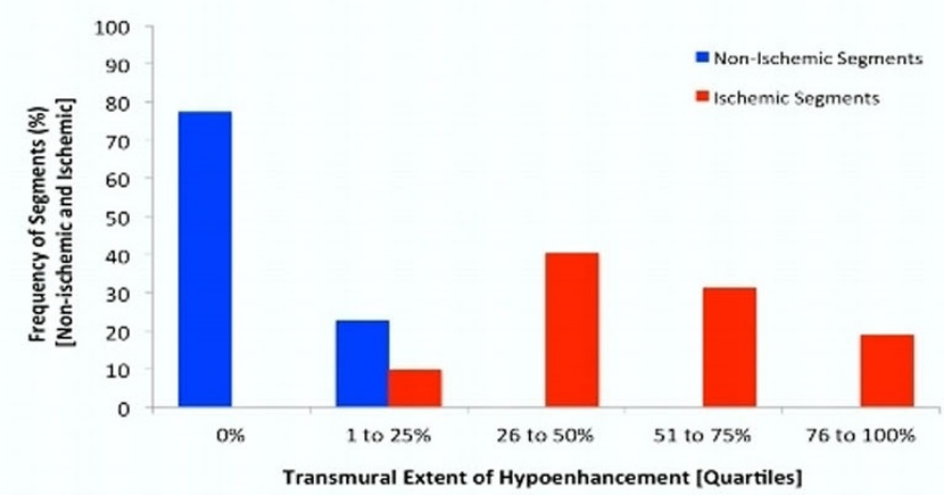

Figure 1 Results of different semi-quantitative parameters (Myocardial Enhancement Time, Persistence of Hypoenhancement, Transmural Extent of Hypoenhancement) derived from the visual interpretation of Stress-Perfusion CMR. The frequency of non-ischemic and ischemic segments in each group is expressed as percent of the total number of non-ischemic or ischemic segments, respectively. A) Frequency of non-ischemic (blue) segments and ischemic (red) segments according to groups of different Myocardial Enhancement Times ( $x$-axis) in frames. B) Frequency of non-ischemic (blue) segments and ischemic (red) segments according to the Persistence of Hypoenhancement ( $x$-axis) in frames. C) Frequency of non-ischemic (blue) segments and ischemic (red) segments according to the Transmural Extent of Hypoenhancement ( $x$-axis) in quartiles. 


\section{Author details}

'Duke Cardiovascular Magnetic Resonance Center, Duke University, Durham,

NC, USA. ${ }^{2}$ Siemens Healthcare, Chapel Hill, NC, USA.

Published: 30 January 2013

doi:10.1186/1532-429X-15-S1-P210

Cite this article as: Jensen et al: Assessment of semi-quantitative parameters for visual interpretation of stress-perfusion CMR in

obstructive coronary artery disease. Journal of Cardiovascular Magnetic

Resonance 2013 15(Suppl 1):P210.

Submit your next manuscript to BioMed Central and take full advantage of:

- Convenient online submission

- Thorough peer review

- No space constraints or color figure charges

- Immediate publication on acceptance

- Inclusion in PubMed, CAS, Scopus and Google Scholar

- Research which is freely available for redistribution

Submit your manuscript at 